\title{
The High-redshift Universe with Spitzer
}

Maruša Bradač $\check{1}^{1,2}$

${ }^{1}$ Department of Physics, University of California, Davis, CA 95616, USA

When did galaxies start forming stars? What is the role of distant galaxies in galaxy formation models and the epoch of reionization? What are the conditions in typical star-forming galaxies at $z \gtrsim 4$ ? Why is galaxy evolution dependent on environment? The Spitzer Space Telescope has been a crucial tool for addressing these questions. Accurate knowledge of stellar masses, ages, and star formation rates (SFRs) requires measuring rest-frame optical (and UV) light, which only Spitzer can probe at high-redshift for a sufficiently large sample of typical galaxies. Many of these science goals are the main science drivers for James Webb Space Telescope, and Spitzer afforded us their first exploration.

Very little was known about the high-redshift universe when the Spitzer Space Telescope [1] was being planned in the 90's. The then optimistic science cases that were put forward were predicting Spitzer could observe ordinary galaxies at $z \sim 5$ [2, Chapter 15]. Given that the highestredshift galaxy discovered at that time was $z_{\mathrm{spec}}=4.7$ [3], this was quite an extraordinary claim. It was not until 1998 that the redshift five barrier was broken [4]. While spectroscopic searches with Lyman- $\alpha$ were proposed in the 1960's, and radio galaxies were discovered beyond $z>3$ in the late 1980's [5, 6], it was the powerful new photometric method [7] to search for "Lyman Break Galaxies" (LBGs) that opened a new window for efficient discoveries of high-redshift galaxies. This meant that high redshift galaxies were being discovered in large numbers, in particular with 
Hubble Space Telescope (HST).

Spitzer, after its launch, soon followed these discoveries. While ordinary galaxies at $z \gtrsim 5$ (i.e., those with characteristic luminosities $L^{*}$ ) needed large time investment before they could be detected, there are now many surveys that are deep enough to observe those galaxies and study their properties (Fig. 11). Among the first studies of $z>5$ galaxies with Spitzer were observations with Spitzer's Infrared Array Camera (IRAC, 8) of objects gravitationally lensed by foreground clusters. This allowed early detection of galaxies at $z=6.7$ [9] and $z=6.56$ [10]. This was only possible through the considerable magnification of these galaxies afforded by the presence of the massive cluster. Gravitational lensing resulted in a significant increase in depth and resolution, a feature that Spitzer has continued to benefit from ever since. Larger samples at $z \gtrsim 5$ were discovered concurrently by virtue of deep observations of the HUDF [11, 12]. The exposure time needed to reach the required depth was many hours and for the first time this was achieved with Spitzer Great Observatories Origins Deep Survey (GOODS, PI Dickinson) program for a large enough area.

The present landscape has changed considerably since early Spitzer observations. While $H S T$ is still the prime telescope to search for and identify the highest redshift galaxies, Spitzer has allowed us to study these galaxies in detail. For cosmic dawn sources (i.e., galaxies at $z>6$ ) Spitzer became a key telescope not only to help establish redshifts, but also in the study of stellar masses, star formation rates, optical emission line strengths, and the identification of old stellar populations. 
For intermediate redshift sources ( $4<z<6$ in this review), Spitzer has also been crucial in robustly determining their stellar masses. In addition, although not discussed in detail here (but see Comment to Nature Astronomy by Daniela Calzetti), at $z<2$ observations with the Multiband Imaging Photometer for Spitzer (MIPS, 13) have been central to measurements of cosmic star formation history. Finally, Spitzer was not only succesful in finding high redshift galaxy clusters $(z>1)$, but also in studying the stellar properties of their cluster members. In this review, we give a brief overview of how Spitzer has provided measurements confirming our expectations, as well as of new puzzles that are changing the paradigm of galaxy formation at high redshifts.

\section{Cosmic Dawn}

Tracing star formation to the earliest times has been a long-standing goal of extragalactic astronomy. In particular, studying the onset of star formation is of importance not only for galaxy formation models, but also for studies of the early universe. The cosmic Dark Ages - when the Universe was filled with neutral hydrogen that was opaque to ultraviolet (UV) light - are thought to have ended around 500 million years after the Big Bang, when early light sources produced enough energetic photons to ionize the neutral hydrogen [14]. This phase is referred to as the epoch of reionization, and is also the era of the formation of the first galaxies. It is now clear that the process was completed by $z \sim 6[15,16,17]$. However, a direct link between early light sources and reionization requires a detailed understanding of when and how galaxies first formed and built up their stellar content. 
Spitzer played (and is continuing to do so with the archival data) a unique role in advancing our understanding of the formation and evolution of galaxies at $z \gtrsim 6$. Deep observations at $3.6 \mu \mathrm{m}$ and $4.5 \mu \mathrm{m}$ with IRAC probe rest-frame optical properties of these galaxies, hence Spitzer data is critical for age (Fig. 2) and stellar mass determination at high-redshift. While observations with HST measure UV light emitted by young stars, Spitzer IRAC measures the rest-frame optical light from long-lived stars in galaxies. In addition, light from some of the most prominent rest-frame optical emission lines (e.g., [O III]4959, 5007 $+\mathrm{H} \beta$ ) enter Spitzer $3.6 \mu \mathrm{m}$ and $4.5 \mu \mathrm{m}$ bands at $z>6$, allowing us to measure their contributions.

Deep observations with Spitzer were first undertaken in the HST deep fields. Of the most ambitious projects with Spitzer designed (in part) for the purposes of observing high-redshift ( $z>$ 6) galaxies was the deep IRAC imaging of the HUDF field (IRAC Ultradeep Field IUDF program PI Labbé, 18). Many large Spitzer surveys followed, the deepest blank-field survey to date being GREATS [19], with a near-homogeneous observing depth of 200 hours over $\sim 200 \operatorname{arcmin}^{2}$ (see also Fig. 3). Results quickly revealed that the galaxies at these redshifts have different rest-frame optical properties from their lower-redshift counterparts.

From the very beginning Spitzer benefited greatly from the magnification due to gravitational lensing. While lensing decreases the effective area surveyed, it more than compensates by increasing the depth and resolution (Fig. 1). Many surveys have recently been executed with this in mind. Among the largest are the Cluster Lensing And Supernova survey with Hubble (CLASH; 20), Hubble Frontier Fields (HFF; 21), Reionization Lensing Cluster Survey (RELICS; 22). Many 
targets for these surveys have been selected from Massive Cluster Survey (MACS; 23, 24). Each of these surveys has its Spitzer counterpart with IRAC Lensing Survey (PI Egami), iCLASH (PI Bouwens, 25), SURFSUP (PI Bradač, 26), SHFF (PI Capak), SRELICS (PI Bradac, 27). These surveys have delivered many interesting results, especially at the lower end of the galaxy stellar mass function. For the largest among them, catalogs have been published as well and stellar properties investigated [28, 29, 30, 31, 32, 33]. Perhaps the highest-redshift galaxy so far detected by Spitzer to date is a lensed galaxy. In [34] the authors report a detection of MACS0647-JD $z \sim 11$ galaxy candidate strongly lensed by a cluster. It is likely a massive and rapidly star-forming galaxy.

Already the first samples of galaxies that were detected in Spitzer/IRAC images (e.g., 18, 35, 36, 37) showed stellar masses in the range from $\sim 10^{9}$ to $\sim 10^{11} M_{\odot}$. Galaxies with stellar masses comparable to the Milky Way masses seemed surprisingly large for a universe younger than 1 Gyr old (e.g., 11). However, it quickly became clear that at least for some of these galaxies their masses have likely been overestimated, due to their IRAC fluxes being boosted by strong nebular emission

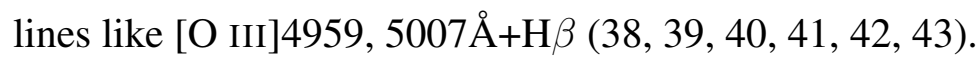

Spitzer broad-band photometry can provide indirect measurements of the nebular lines. For example, Spitzer/IRAC colors can be used to measure strengths of [O III]+H $\beta$ lines for $6.6 \lesssim z \lesssim$ 6.9 galaxies, for which these lines are expected to fall in the $3.6 \mu \mathrm{m}$ band while the $4.5 \mu \mathrm{m}$ band is relatively free of line contamination (Fig. 4, 41, 44, 45, 46). Similarly, [O III]+H $\beta$ lines land in $4.5 \mu \mathrm{m}$ for galaxies at $7 \lesssim z \lesssim 9[19,27,27,43,47,48]$, while the $3.6 \mu \mathrm{m}$ band remains relatively free of strong emission lines. 
The results from these works show that we are now faced with a puzzle. Taken the Spitzer colors at face value, the line strengths required to fit the data for many of these galaxies is extremely high, with rest-frame equivalent widths $E W>2000 \AA$. But there is also a degeneracy in these measurements, as the color can also be boosted by an older stellar population in the form of a Balmer break and/or dust [49]. Thus, rest-frame optical emission lines are likely not to be the sole cause of excess flux in the rest-frame optical bands. Unfortunately, spectroscopic observations of rest-frame optical emission lines of the highest redshift galaxies are out of reach for the current instruments. The sensitivity of the InfraRed Spectrograph (IRS) on Spitzer [50] was mostly limited to observations of $z<3$ IR luminous galaxies [51, 52], and we will have to wait for JWST to allow us to perform efficient spectroscopic follow-up in the rest-frame optical.

However, with Spitzer we are already able to study some exceptional cases where we can mitigate or break these degeneracies. In particular, when spectroscopic redshifts are known, we can break the degeneracy by considering particular redshift ranges where lines are shifted out of the filters and/or by adding external data. For example, one can, in principle, use Lyman- $\alpha$ fluxes to estimate the nebular emission line contribution [42] or can constrain the amount of dust using measurements using ALMA [53, 54].

The best case where the degeneracy has been lifted is a $z=9.1$ galaxy MACS1149-JD behind the cluster MACS J1149.5+2223. MACS1149-JD was originally discovered in HST and shallow $4.5 \mu \mathrm{m}$ Spitzer data in [55]. It was later detected in both, $3.6 \mu \mathrm{m}$ and $4.5 \mu \mathrm{m}$, Spitzer bands using deeper data (Fig. 5, 26, 46, 56) and its redshift was spectroscopically measured by 
[53]. For this galaxy the nebular emission lines are redshifted out of these Spitzer bands, yet it has a strong color excess. In addition, the cold dust content of the galaxy was constrained to be modest from observations taken with ALMA, making at least cold dust an unlikely cause of the red Spitzer color [53]. It is therefore highly likely the old ( $\sim 300 \mathrm{Myr})$ stellar population is causing the red rest-frame optical color (46, 53, 57). This is surprising, given the galaxies would need to start forming significant amounts of stars shortly after the Big Bang. At $z=9.1$, when the Universe is only $\lesssim 550 \mathrm{Myr}$ old, the presence of a strong Balmer break can thus provide the timing of the first star formation. In the case of MACS1149-JD the dominant stellar component formed about 250 million years after the Big Bang, corresponding to a redshift of about 15.

There are several other objects with Spitzer detections at $z \gtrsim 9$ where this experiment can be repeated. The highest spectroscopically confirmed galaxy detected by Spitzer (GN-z11, 58) does not show a red color. There are others that do, e.g. GN-z10-3 and GN-z9-1 [59] and Abell17631434 [27]. They all have $4.5 \mu \mathrm{m}$ excesses that are consistent with an evolved stellar population, but unfortunately they currently lack spectroscopic confirmations. If their redshifts are confirmed, it is likely that a re-analysis of these galaxies would indicate that their star formation occurred within 300Myr of the Big Bang.

While the sample is still small, observations of galaxies at $z>6$ with Spitzer nonetheless show that galaxies have unexpected properties. A large fraction of high-redshift galaxies have either unusually strong nebular emission lines, pronounced Balmer breaks indicating old stellar populations, or the large amounts of dust. All three possibilities are difficult to reconcile given the 
age of the Universe. E.g., old stellar population in MACS1149-JD puts formation redshift of the majority of stellar population $\left(\sim 10^{9} M_{\odot}\right)$ at $z \sim 15$ which is high given that simulations predict first stars only started forming at $z \sim 30$ [60]. Other objects are routinely showing large restframe equivalent widths ( $E W \sim 1000 \AA$ ) of nebular emission lines, these have not been observed at lower redshifts except for in some extreme cases (e.g., 61, 62). IRAC color excess could also be explained by the presence of dust, but it is difficult to produce a significant amount of dust needed to explain the observations [49]. While more precise answers await the James Webb Space Telescope (JWST), it is clear even at present, the star formation models used in simulations at the highest redshift are being constrained as a result of observations by Spitzer.

\section{Cosmic Morning}

Studies of intermediate redshift $(4<z<6)$ have also thrived thanks to Spitzer observations. One of the main diagnostics in galaxy evolution models is the evolution of the Star Formation Rate Density (SFRD, 63). However, robust determination of dust attenuation is essential to transform FUV luminosity densities into total SFRDs. Prior to Spitzer observations star formation history was determined out to $z \sim 4$ using mostly HST data [64, 65, 66]. It was only after Spitzer data (along with Herschel) was obtained, that the history of cosmic star formation could be robustly measured, and the finding that the majority of star formation density comes from dust-obscured sources was established (63 and references therein). MIPS also played an important role in these discoveries at lower redshifts (e.g., [67, 68]). 
The true power of Spitzer comes with robust measurements of stellar masses at these redshifts. The stellar-mass function measurements were first undertaken for SCANDELS, SCOSMOS, SPLASH, and UDF fields (e.g., 69, 70, 71, 72, 73, 74). But the largest of surveys to explore this are The Spitzer Matching Survey of the UltraVISTA Ultra-deep Stripes (SMUVS, PI Caputi, 75, 76) and Euclid/WFIRST Spitzer Legacy Survey (Moneti et al. in prep.). With SMUVS, a large fraction of galaxies ( $\gtrsim 50 \%$ ) which were previously detected in the optical, were, for the first time, also detected with Spitzer. This allowed for a precise measurement of the stellar mass function. The latest results indicate that massive and intermediate-mass galaxies have different evolutionary paths in the early universe [77].

One of the key properties to describe galaxy growth is the ratio between star formation rate (SFR) and stellar mass $\left(M_{*}\right)$, also known as specific star formation rate $\left(\mathrm{sSFR}=\mathrm{SFR} / M_{*} ; 63,78\right)$. This ratio depends heavily on good estimates of both stellar masses and SFR. In addition, sSFR is particularly well-determined in the lensing fields, as its value is independent of magnification, and yet gravitational lensing allows us to study sub- $L^{*}$ galaxies at high redshift [31]. Its evolution with redshift is one of the key questions in galaxy formation studies and we are still unclear as to exactly how sSFR evolves. While pioneering studies predicted a constant sSFR at high redshifts [79, 80], the later results, which included nebular emission lines in SED fitting [81] and better Spitzer data find an increase at $4<z<6$ and beyond (e.g., 31, 71, 73, 82, 83, 84, 85, 86, 87).

With Spitzer also SFR using $\mathrm{H} \alpha$ was investigated in detail for the first time at $4<z<5$, which is regarded as one of the most reliable among the easily accessible nebular SFR tracers 
(e.g..88). Just like with [O III], at intermediate redshifts there are ranges where emission line strength of $\mathrm{H} \alpha$ can be measured with Spitzer (44, Fig. 4). One of the caveats is that $\mathrm{H} \alpha$ is blended with N II. Still, this has been used to indirectly measure the $\mathrm{H} \alpha$ strength at $3.9 \lesssim z \lesssim 4.9$ and deduce the star formation rates [73, 76, 87, 89, 90, 91, 92]. More recently, in [93] authors measured burstiness of star formation by comparing ultra-violet, $\mathrm{H} \alpha$ luminosity, and $\mathrm{H} \alpha$ equivalent-width of $z \sim 4-5$ main sequence galaxies, indicating that for at least half of their sample the star formation history is not smooth. Access to $\mathrm{H} \alpha$ also allows modelling of Lyman-continuum photon

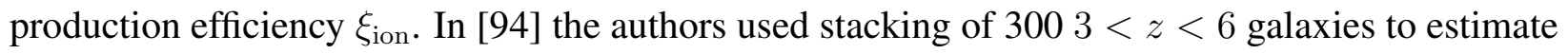
$\mathrm{H} \alpha$ equivalent widths and found that $\xi_{\text {ion }}$ is not strongly dependent on luminosity and is thus similar to the values derived for brighter galaxies. This has important implications, in particular for establishing that faint galaxies are able to produce the Lyman-continuum photons needed for cosmic reionization.

While Spitzer has improved our knowledge of stellar masses and SFRs considerably, several issues remain. In particular, the evolution of sSFR is still not a completely solved problem. The situation will likely improve soon with the data taken from Spitzer in the last year, as well as in the future with missions like JWST, Euclid and the Wide-field Infrared Survey Telescope (WFIRST).

\section{Cosmic Dusk}

The present epoch is one of a rapid decline in the global star formation rate, but clusters experience an evolution in star formation activity over this time that is even stronger than in the field. Identi- 
fying the processes that trigger and terminate star formation in cluster galaxies (e.g. ram-pressure stripping, starvation, merging, harassment; 95, 96), and contrasting them to those operating in the field is key to understanding the causes of the general decline. Furthermore, studying clusters at earlier times provides new constraints on both the evolution of the cluster abundance and the evolution of early-type galaxies over a substantial look-back time [97]. Spitzer plays a large role in identifying both high-redshift $(z>1)$ clusters and protoclusters. Red-sequence overdensities and color searches can efficiently be done with Spitzer and have been utilized in both high-redshift cluster and protocluster searches. In particular, the inclusion of Spitzer data dramatically increases the accuracy/precision of photometric redshifts at $z>1$, which is crucial for finding structures and to robustly estimate the environment (e.g., 98).

Early on, Spitzer helped in discoveries of the protoclusters. In [99] the authors present the discovery of a $z=5.7$ protocluster that dates back to $1 \mathrm{Gyr}$ after the Big Bang (Fig. 6). The field contains a luminous quasar as well as a large galaxy rich in molecular gas. The Clusters Around Radio-Loud AGN (CARLA) survey used this fact, as CARLA targeted radio-loud quasars with Spitzer at $z>1.3$, discovering several proto-clusters surrounding them [100, 101]. Spitzer data is used to improve redshift information, as well as to estimate stellar masses, SFR and ages as these are the only bands available that probe the optical/NIR rest-frame for high-redshift protoclusters.

Spitzer played an even more prominent role in finding the highest redshift galaxy clusters. Shallow yet wide surveys, in particular the IRAC Shallow Survey, enabled detections of many high-redshift clusters (102, 103, 104). More discoveries were made in the Spitzer Wide-Area 
Infrared Extragalactic (SWIRE) Survey using a simple color excess in Spitzer bands [105]. The large area is needed as clusters of galaxies are extremely rare, and one requires such a survey to find the most massive examples (using numbers from [106] $\sim 6 \mathrm{deg}^{2}$ are needed to detect one high-redshift $z \gtrsim 1$, massive $M>10^{14} M_{\odot}$ cluster; Fig. 11.

The Spitzer Adaptation of the Red-sequence Cluster Survey (SPARCS; 107, 108; Fig. 6) was the first to perform a comprehensive study of $z>1$ galaxy clusters with all IRAC and MIPS bands. At $z=1.5$, the 1.6 micron bump [109], due to infrared emission of stars, is shifted into Spitzer $4.5 \mu \mathrm{m}$ band, making it a useful tool to detect cluster members. Finally, surveys like IRAC Distant Cluster Survey [110], MaDCoWS (using WISE, but utilizing Spitzer for confirmation; 106) and Spitzer South Pole Telescope Deep Field [111, 112] have also detected many rich clusters at $z>1$. Even more importantly, both IRAC and MIPS enabled the study the stellar properties of cluster members [113, 114]. The main conclusion from these surveys is that while low-redshift clusters are considered mostly star formation graveyards, at the earlier times $(z>1)$, galaxies in galaxy clusters were star forming and active [113]. This is thus the star-forming epoch of galaxy clusters, the study of which was enabled by Spitzer.

\section{Thank You Spitzer}

When Spitzer was first planned, nobody was expecting it to do the groundbreaking discoveries at the cosmic dawn. This was mostly due to the mirror size, yet the $85 \mathrm{~cm}$ diameter mirror (only slightly larger than a wine-barrel) has surpassed a lot of predictions. It was meant to explore "the 
Old, the Cold, and the Dusty", and yet it also explored the starry, the many, and the first. Spitzer let us study early star formation in the first galaxies at the epoch of reionization, it delivered new insights on star formation at high-redshift, and allowed us to find and study the earliest galaxy clusters and protoclusters. While the next space telescope (JWST) will revolutionize these fields in many ways, it will not surpass Spitzer in its ability to do survey science thanks to Spitzer's combination of a large field of view, excellent sensitivity, and long life. So long Spitzer, and thanks for all the photons.

Acknowledgements Based on observations made with the Spitzer Space Telescope, which is operated by the Jet Propulsion Laboratory, California Institute of Technology under a contract with NASA. Support for this work was provided by NASA through ADAP grant 80NSSC18K0945, NSF grant AST 1815458 and through an award issued by JPL/Caltech. The author would like to thank Brian Lemaux and Victoria Strait for their help with the manuscript.

Financial Interest The authors declare no competing financial interests.

Correspondence Correspondence should be addressed to M.B.

1. Werner, M. W. et al. The Spitzer Space Telescope Mission. Astrophys. J. Suppl. 154, 1-9 (2004). astro-ph/0406223.

2. Werner, M. \& Eisenhardt, P. More Things in the Heavens: How Infrared Astronomy is Expanding our View of the Universe (Princeton University Press, 2019). 
3. Schneider, D. P., Schmidt, M. \& Gunn, J. E. PC 1158+4635: an Optically Selected Quasar with a Redshift of 4.73. Astron. J. 98, 1951 (1989).

4. Dey, A., Spinrad, H., Stern, D., Graham, J. R. \& Chaffee, F. H. A Galaxy at z=5.34. Astrophys. J. Lett. 498, L93 (1998). arXiv:astro-ph/9803137.

5. Lilly, S. J. Discovery of a Radio Galaxy at a Redshift of 3.395. Astrophys. J. 333, 161 (1988).

6. Chambers, K. C., Miley, G. K. \& van Breugel, W. J. M. 4C 41.17: A Radio Galaxy at a Redshift of 3.8. Astrophys. J. 363, 21 (1990).

7. Steidel, C. C., Giavalisco, M., Pettini, M., Dickinson, M. \& Adelberger, K. L. Spectroscopic Confirmation of a Population of Normal Star-forming Galaxies at Redshifts Z $>$ 3. Astrophys. J. Lett. 462, L17+ (1996). arXiv:astro-ph/9602024.

8. Fazio, G. G. et al. The Infrared Array Camera (IRAC) for the Spitzer Space Telescope. Astrophys. J. Suppl. 154, 10-17 (2004). astro-ph/0 405616.

9. Egami, E. et al. Spitzer and Hubble Space Telescope Constraints on the Physical Properties of the $z \sim 7$ Galaxy Strongly Lensed by A2218. Astrophys. J. Lett. 618, L5-L8 (2005).

10. Chary, R.-R., Stern, D. \& Eisenhardt, P. SpitzerConstraints on the z=6.56 Galaxy Lensed by Abell 370. The Astrophysical Journal 635, L5-L8 (2005).

11. Yan, H. et al. Rest-Frame Ultraviolet-to-Optical Properties of Galaxies at $\mathrm{z} \sim 6$ and $\mathrm{z} \sim 5$ in the Hubble Ultra Deep Field: From Hubble to Spitzer. Astrophys. J. 634, 109-127 (2005). astro-ph/0507673. 
12. Eyles, L. P. et al. Spitzer imaging of i'-drop galaxies: old stars at z 6. Mon. Not. R. Astron. Soc. 364, 443-454 (2005). astro-ph/0502385.

13. Rieke, G. H. et al. The Multiband Imaging Photometer for Spitzer (MIPS). Astrophys. J. Suppl. 154, 25-29 (2004).

14. Planck Collaboration et al. Planck 2015 results. XIII. Cosmological parameters. Astron. Astrophys. 594, A13 (2016). 1502.01589.

15. Fan, X., Carilli, C. L. \& Keating, B. Observational Constraints on Cosmic Reionization. Annu. Rev. Astron. Astrophys. 44, 415-462 (2006). astro-ph/ 0602375.

16. Robertson, B. E., Ellis, R. S., Furlanetto, S. R. \& Dunlop, J. S. Cosmic Reionization and Early Star-forming Galaxies: A Joint Analysis of New Constraints from Planck and the Hubble Space Telescope. Astrophys. J. Lett. 802, L19 (2015). 1502 .02024.

17. Mason, C. A. et al. Inferences on the timeline of reionization at $\mathrm{z} \sim 8$ from the KMOS Lens-Amplified Spectroscopic Survey. Mon. Not. R. Astron. Soc. 485, 3947-3969 (2019). 1901.11045 ,

18. Labbé, I. et al. Ultradeep Infrared Array Camera Observations of Sub-L* $\mathrm{z} \sim 7$ and $\mathrm{z} \sim$ 8 Galaxies in the Hubble Ultra Deep Field: the Contribution of Low-Luminosity Galaxies to the Stellar Mass Density and Reionization. Astrophys. J. Lett. 708, L26-L31 (2010). 0910.0838

19. Stefanon, M. et al. The Brightest $\mathrm{z} \gtrsim 8$ Galaxies over the COSMOS UltraVISTA Field. Astrophys. J. 883, 99 (2019). 1902.10713. 
20. Postman, M. et al. The Cluster Lensing and Supernova Survey with Hubble: An Overview. Astrophys. J. Suppl. 199, 25 (2012). 1106.3328.

21. Lotz, J. M. et al. The Frontier Fields: Survey Design and Initial Results. Astrophys. J. 837, 97 (2017).

22. Coe, D. et al. RELICS: Reionization Lensing Cluster Survey. Astrophys. J. 884, 85 (2019).

\subsection{2.}

23. Ebeling, H. et al. A Complete Sample of 12 Very X-Ray Luminous Galaxy Clusters at $z>0.5$. Astrophys. J. Lett. 661, L33-L36 (2007). arXiv:astro-ph/ 0703394.

24. Repp, A. \& Ebeling, H. Science from a glimpse: Hubble SNAPshot observations of massive galaxy clusters. Mon. Not. R. Astron. Soc. 479, 844-864 (2018). 1706.01263.

25. Bouwens, R. J. et al. A Census of Star-forming Galaxies in the $z \sim 9-10$ Universe based on HST+Spitzer Observations over 19 Clash Clusters: Three Candidate $Z \sim 9-10$ Galaxies and Improved Constraints on the Star Formation Rate Density at $z \sim 9.2$. Astrophys. J. 795, 126 (2014). 1211.2230.

26. Bradač, M. et al. Spitzer Ultra Faint SUrvey Program (SURFS UP). I. An Overview. Astrophys. J. 785, 108 (2014). 1402.2352.

27. Strait, V. et al. Stellar properties of $\mathrm{z} \gtrsim 8$ galaxies in the reionization lensing cluster survey. The Astrophysical Journal 888, 124 (2020). 
28. Merlin, E. et al. The ASTRODEEP Frontier Fields catalogues. I. Multiwavelength photometry of Abell-2744 and MACS-J0416. Astron. Astrophys. 590, A30 (2016). 1603.02460.

29. Castellano, M. et al. The ASTRODEEP Frontier Fields catalogues. II. Photometric redshifts and rest frame properties in Abell-2744 and MACS-J0416. Astron. Astrophys. 590, A31 (2016). 1603.02461 .

30. Di Criscienzo, M. et al. The ASTRODEEP Frontier Fields catalogues. III. Multiwavelength photometry and rest-frame properties of MACS-J0717 and MACS-J1149. Astron. Astrophys. 607, A30 (2017). 1706.03790 .

31. Santini, P. et al. The Star Formation Main Sequence in the Hubble Space Telescope Frontier Fields. Astrophys. J. 847, 76 (2017). 1706.07059.

32. Shipley, H. V. et al. HFF-DeepSpace Photometric Catalogs of the 12 Hubble Frontier Fields, Clusters, and Parallels: Photometry, Photometric Redshifts, and Stellar Masses. Astrophys. J. Suppl. 235, 14 (2018). 1801.09734.

33. Bradač, M. et al. Hubble Frontier Field photometric catalogues of Abell 370 and RXC J2248.7-4431: multiwavelength photometry, photometric redshifts, and stellar properties. Mon. Not. R. Astron. Soc. 489, 99-107 (2019). 1906.01725.

34. Lam, D. et al. Detection of a Lensed $\mathrm{z} \approx 11$ Galaxy in the Rest-Optical with Spitzer/IRAC and the Inferred SFR, Stellar Mass, and Physical Size. arXiv e-prints arXiv:1903.08177 (2019). 1903.08177. 
35. Yan, H. et al. The Stellar Masses and Star Formation Histories of Galaxies at $z \sim 6$ : Constraints from Spitzer Observations in the Great Observatories Origins Deep Survey. The Astrophysical Journal 651, 24 (2006).

36. Capak, P. et al. Spectroscopy of Luminous $z>7$ Galaxy Candidates and Sources of Contamination in $z>7$ Galaxy Searches. Astrophys. J. 730, 68 (2011). 0910.0444.

37. Labbe, I. et al. The Spectral Energy Distributions of $z \sim 8$ Galaxies from the IRAC Ultra Deep Fields: Emission Lines, Stellar Masses, and Specific Star Formation Rates at 650 Myr. Astrophys. J. Lett. 777, L19 (2013). 1209.3037.

38. Finkelstein, S. L. et al. A galaxy rapidly forming stars 700 million years after the Big Bang at redshift 7.51. Nature 502, 524-527 (2013).

39. Smit, R. et al. EVIDENCE FOR UBIQUITOUS HIGH-EQUIVALENT-WIDTH NEBULAR EMISSION IN $z \sim 7$ GALAXIES: TOWARD A CLEAN MEASUREMENT OF THE SPECIFIC STAR-FORMATION RATE USING A SAMPLE OF BRIGHT, MAGNIFIED GALAXIES. The Astrophysical Journal 784, 58 (2014).

40. De Barros, S., Schaerer, D. \& Stark, D. P. Properties of z 3-6 Lyman break galaxies. A\&A 563, A81 (2014).

41. Roberts-Borsani, G. W. et al. $\mathrm{z} \gtrsim 7$ Galaxies with Red Spitzer/IRAC [3.6]-[4.5] Colors in the Full CANDELS Data Set: The Brightest-Known Galaxies at z 7-9 and a Probable Spectroscopic Confirmation at $\mathrm{z}=7.48$. Astrophys. J. 823, 143 (2016). 1506.00854. 
42. Jiang, L. et al. Physical Properties of Spectroscopically Confirmed Galaxies at $\mathrm{z} \geq 6$. III. Stellar Populations from SED Modeling with Secure Ly $\alpha$ Emission and Redshifts. Astrophys. J. 816, 16 (2016). 1511.01519.

43. De Barros, S. et al. The GREATS H $\beta+[\mathrm{O}$ III] luminosity function and galaxy properties at $z \sim 8$ : walking the way of JWST. Mon. Not. R. Astron. Soc. 489, 2355-2366 (2019). 1903.09649 ,

44. Shim, H. et al. $z \sim 4 \mathrm{H} \alpha$ Emitters in the Great Observatories Origins Deep Survey: Tracing the Dominant Mode for Growth of Galaxies. Astrophys. J. 738, 69 (2011). 1103.4124.

45. Smit, R. et al. High-precision Photometric Redshifts from Spitzer/IRAC: Extreme [3.6] [4.5] Colors Identify Galaxies in the Redshift Range $z \sim 6.6-6.9$. Astrophys. J. 801, 122 (2015). 1412.0663 .

46. Huang, K.-H. et al. Detection of Lyman-alpha Emission from a Triply Imaged $\mathrm{z}=6.85$ Galaxy behind MACS J2129.4-0741. Astrophys. J. Lett. 823, L14 (2016). 1605.05771.

47. Laporte, N. et al. The first Frontier Fields cluster: $4.5 \mu \mathrm{m}$ excess in a $z \sim 8$ galaxy candidate in Abell 2744. Astron. Astrophys. 562, L8 (2014). 1401.8263.

48. Bridge, J. S. et al. The Super Eight Galaxies: Properties of a Sample of Very Bright Galaxies at $7<z<8$. Astrophys. J. 882, 42 (2019). 1907.05512.

49. Katz, H., Laporte, N., Ellis, R. S., Devriendt, J. \& Slyz, A. Probing cosmic dawn: modelling the assembly history, SEDs, and dust content of selected $\mathrm{z} \sim 9$ galaxies. Mon. Not. R. Astron. Soc. 484, 4054-4068 (2019). 1809.07210. 
50. Houck, J. R. et al. The Infrared Spectrograph (IRS) on the Spitzer Space Telescope. Astrophys. J. Suppl. 154, 18-24 (2004). astro-ph/0406167.

51. Teplitz, H. I. et al. Measuring PAH Emission in Ultradeep Spitzer IRS Spectroscopy of High-Redshift IR-Luminous Galaxies. Astrophys. J. 659, 941-949 (2007). astro-ph/ 0701409 .

52. Pope, A. et al. Mid-Infrared Spectral Diagnosis of Submillimeter Galaxies. Astrophys. J. 675, 1171-1193 (2008). 0711.1553 .

53. Hashimoto, T. et al. The onset of star formation 250 million years after the Big Bang. Nature 557, 392-395 (2018). 1805.05966.

54. Laporte, N. et al. Dust in the Reionization Era: ALMA Observations of a $\mathrm{z}=8.38$ Gravitationally Lensed Galaxy. Astrophys. J. Lett. 837, L21 (2017). 1703.02039.

55. Zheng, W. et al. A magnified young galaxy from about 500 million years after the Big Bang. Nature 489, 406-408 (2012). 1204 . 2305.

56. Zheng, W. et al. Young Galaxy Candidates in the Hubble Frontier Fields. IV. MACS J1149.5+2223. Astrophys. J. 836, 210 (2017). 1701.08484.

57. Hoag, A. et al. Spectroscopic confirmation of an ultra-faint galaxy at the epoch of reionization. Nature Astronomy 1, 0091 (2017). 1704.02970.

58. Oesch, P. A. et al. A Remarkably Luminous Galaxy at $\mathrm{z}=11.1$ Measured with Hubble Space Telescope Grism Spectroscopy. Astrophys. J. 819, 129 (2016). 1603.00461. 
59. Oesch, P. A. et al. The Most Luminous z $\sim 9-10$ Galaxy Candidates Yet Found: The Luminosity Function, Cosmic Star-formation Rate, and the First Mass Density Estimate at 500 Myr. Astrophys. J. 786, 108 (2014). 1309.2280.

60. Dayal, P. \& Ferrara, A. Early galaxy formation and its large-scale effects. Phys. Rep. 780, 1-64 (2018). 1809.09136.

61. Du, X. et al. Searching for $z>6.5$ Analogs Near the Peak of Cosmic Star Formation. Astrophys. J. 890, 65 (2020). 1910 . 11877.

62. Li, Z. \& Malkan, M. A. Extreme [O III] Emitters at $z \sim 0.5$. Astrophys. J. 860, 83 (2018). 1804.11309 .

63. Madau, P. \& Dickinson, M. Cosmic Star-Formation History. Annu. Rev. Astron. Astrophys. 52, 415-486 (2014). 1403.0007.

64. Madau, P. et al. High-redshift galaxies in the Hubble Deep Field: colour selection and star formation history to z 4. Mon. Not. R. Astron. Soc. 283, 1388-1404 (1996). astro-ph/ 9607172

65. Madau, P., Pozzetti, L. \& Dickinson, M. The Star Formation History of Field Galaxies. Astrophys. J. 498, 106-116 (1998). astro-ph/9708220.

66. Lilly, S. J., Le Fevre, O., Hammer, F. \& Crampton, D. The Canada-France Redshift Survey: The Luminosity Density and Star Formation History of the Universe to Z approximately 1. Astrophys. J. Lett. 460, L1 (1996). astro-ph/9601050. 
67. Le Floc'h, E. et al. Infrared Luminosity Functions from the Chandra Deep Field-South: The Spitzer View on the History of Dusty Star Formation at $0 \lesssim z \lesssim 1$. Astrophys. J. 632, 169-190 (2005). astro-ph/ 0506462 .

68. Magnelli, B. et al. Evolution of the dusty infrared luminosity function from $\mathrm{z}=0$ to $\mathrm{z}=2.3$ using observations from Spitzer. Astron. Astrophys. 528, A35 (2011). 1101.2467.

69. Davidzon, I. et al. The COSMOS2015 galaxy stellar mass function . Thirteen billion years of stellar mass assembly in ten snapshots. Astron. Astrophys. 605, A70 (2017). 1701.02734.

70. Song, M. et al. The Evolution of the Galaxy Stellar Mass Function at $\mathrm{z}=4-8$ : A Steepening Low-mass-end Slope with Increasing Redshift. Astrophys. J. 825, 5 (2016). 1507.05636.

71. Grazian, A. et al. The galaxy stellar mass function at $3.5 \leq \mathrm{z} \leq 7.5$ in the CANDELS/UDS, GOODS-South, and HUDF fields. Astron. Astrophys. 575, A96 (2015). 1412.0532.

72. Caputi, K. I. et al. Spitzer Bright, UltraVISTA Faint Sources in COSMOS: The Contribution to the Overall Population of Massive Galaxies at $\mathrm{z}=3-7$. Astrophys. J. 810, 73 (2015).

\subsection{1.}

73. Salmon, B. et al. The Relation between Star Formation Rate and Stellar Mass for Galaxies at $3.5 \leq z \leq 6.5$ in CANDELS. Astrophys. J. 799, 183 (2015). 1407.6012 .

74. Steinhardt, C. L. et al. Star Formation at $4<z<6$ from the Spitzer Large Area Survey with Hyper-Suprime-Cam (SPLASH). Astrophys. J. Lett. 791, L25 (2014). 1407 . 7030.

75. Ashby, M. L. N. et al. Spitzer Matching Survey of the UltraVISTA Ultra-deep Stripes 
(SMUVS): Full-mission IRAC Mosaics and Catalogs. Astrophys. J. Suppl. 237, 39 (2018).

\subsection{0 .}

76. Caputi, K. I. et al. Star Formation in Galaxies at $\mathrm{z} \sim 4-5$ from the SMUVS Survey: A Clear Starburst/Main-sequence Bimodality for $\mathrm{H} \alpha$ Emitters on the SFR-M* Plane. Astrophys. J. 849, 45 (2017). 1705.06179.

77. Deshmukh, S. et al. The Spitzer Matching Survey of the UltraVISTA Ultra-deep Stripes (SMUVS): The Evolution of Dusty and Nondusty Galaxies with Stellar Mass at $\mathrm{z}=2-6$. Astrophys. J. 864, 166 (2018). 1712.03905.

78. Guzmán, R. et al. The Nature of Compact Galaxies in the Hubble Deep Field. II. Spectroscopic Properties and Implications for the Evolution of the Star Formation Rate Density of the Universe1,. Astrophys. J. 489, 559-572 (1997). astro-ph/9704001.

79. Stark, D. P. et al. The Evolutionary History of Lyman Break Galaxies Between Redshift 4 and 6: Observing Successive Generations of Massive Galaxies in Formation. Astrophys. J. 697, 1493-1511 (2009). 0902.2907.

80. Gonzalez, A. H., Papovich, C., Bradač, M. \& Jones, C. Spectroscopic Confirmation of a z = 2.79 Multiply Imaged Luminous Infrared Galaxy Behind the Bullet Cluster. Astrophys. J. 720, 245-251 (2010).

81. Schaerer, D. \& de Barros, S. The impact of nebular emission on the ages of $z \sim 6$ galaxies. Astron. Astrophys. 502, 423-426 (2009). 0905.0866. 
82. Davidzon, I., Ilbert, O., Faisst, A. L., Sparre, M. \& Capak, P. L. An Alternate Approach to Measure Specific Star Formation Rates at $2<z<7$. Astrophys. J. 852, 107 (2018).

\subsection{9.}

83. Tasca, L. A. M. et al. Evidence for major mergers of galaxies at $2 \lesssim z<4$ in the VVDS and VUDS surveys. Astron. Astrophys. 565, A10 (2014). 1303.4400.

84. de Barros, S., Schaerer, D. \& Stark, D. P. Properties of $z \sim 3-6$ Lyman break galaxies. II. Impact of nebular emission at high redshift. Astron. Astrophys. 563, A81 (2014). 1207 . 3663.

85. Duncan, K. et al. The mass evolution of the first galaxies: stellar mass functions and star formation rates at $4<\mathrm{z}<7$ in the CANDELS GOODS-South field. Mon. Not. R. Astron. Soc. 444, 2960-2984 (2014). 1408.2527.

86. González, V. et al. Slow Evolution of the Specific Star Formation Rate at z $>2$ : The Impact of Dust, Emission Lines, and a Rising Star Formation History. Astrophys. J. 781, 34 (2014). 1208.4362 .

87. Stark, D. P. et al. Keck Spectroscopy of $3<z<7$ Faint Lyman Break Galaxies: The Importance of Nebular Emission in Understanding the Specific Star Formation Rate and Stellar Mass Density. Astrophys. J. 763, 129 (2013). 1208 . 3529.

88. Moustakas, J., Kennicutt, R. C., Jr. \& Tremonti, C. A. Optical Star Formation Rate Indicators. Astrophys. J. 642, 775-796 (2006). astro-ph/0511730. 
89. Smit, R. et al. Evidence for Ubiquitous High-equivalent-width Nebular Emission in $z \sim 7$ Galaxies: Toward a Clean Measurement of the Specific Star-formation Rate Using a Sample of Bright, Magnified Galaxies. Astrophys. J. 784, 58 (2014). 1307.5847.

90. Bouwens, R. J. et al. The Lyman-Continuum Photon Production Efficiency $\xi_{\text {ion }}$ of $z \sim 4-5$ Galaxies from IRAC-based $\mathrm{H} \alpha$ Measurements: Implications for the Escape Fraction and Cosmic Reionization. Astrophys. J. 831, 176 (2016). 1511.08504.

91. Mármol-Queraltó, E. et al. The evolution of the equivalent width of the $\mathrm{H} \alpha$ emission line and specific star formation rate in star-forming galaxies at 1 \&lt; z \&lt; 5. Mon. Not. R. Astron. Soc. 460, 3587-3597 (2016). 1511.01911.

92. Faisst, A. L. et al. A Coherent Study of Emission Lines from Broadband Photometry: Specific Star Formation Rates and [O iii]/H $\beta$ Ratio at $3<z<6$. Astrophys. J. 821, 122 (2016). 1601.07173.

93. Faisst, A. L., Capak, P. L., Emami, N., Tacchella, S. \& Larson, K. L. The Recent Burstiness of Star Formation in Galaxies at $z \sim 4.5$ from $\mathrm{H} \alpha$ Measurements. Astrophys. J. 884, 133 (2019). 1909.03076.

94. Lam, D. et al. The mean $\mathrm{H} \alpha \mathrm{EW}$ and Lyman-continuum photon production efficiency for faint z $\approx 4-5$ galaxies. Astron. Astrophys. 627, A164 (2019). 1902.02786.

95. Dressler, A. et al. A Spectroscopic Catalog of 10 Distant Rich Clusters of Galaxies. Astrophys. J. Suppl. 122, 51-80 (1999). arXiv:astro-ph/9901263. 
96. Poggianti, B. M. et al. The Star Formation Histories of Galaxies in Distant Clusters. Astrophys. J. 518, 576-593 (1999). astro-ph/9901264.

97. Rosati, P., Borgani, S. \& Norman, C. The Evolution of X-ray Clusters of Galaxies. Annu. Rev. Astron. Astrophys. 40, 539 (2002).

98. Lemaux, B. C. et al. The VIMOS Ultra-Deep Survey: Emerging from the dark, a massive proto-cluster at z 4.57. Astron. Astrophys. 615, A77 (2018). 1703.10170.

99. Capak, P. L. et al. A massive protocluster of galaxies at a redshift of z 5.3. Nature 470, 233-235 (2011). 1101.3586.

100. Galametz, A. et al. The Mid-infrared Environments of High-redshift Radio Galaxies. Astrophys. J. 749, 169 (2012). 1202.4489.

101. Noirot, G. et al. HST Grism Confirmation of 16 Structures at $1.4<z<2.8$ from the Clusters Around Radio-Loud AGN (CARLA) Survey. Astrophys. J. 859, 38 (2018). 1804.01500.

102. Stanford, S. A. et al. An IR-selected Galaxy Cluster at $\mathrm{z}=1.41$. Astrophys. J. Lett. 634, L129-L132 (2005). astro-ph/0510655.

103. Stanford, S. A. et al. The XMM Cluster Survey: A Massive Galaxy Cluster at $\mathrm{z}=1.45$. Astrophys. J. Lett. 646, L13-L16 (2006). astro-ph/ 0606075.

104. Eisenhardt, P. R. M. et al. Clusters of Galaxies in the First Half of the Universe from the IRAC Shallow Survey. Astrophys. J. 684, 905-932 (2008). 0804 . 4798. 
105. Papovich, C. et al. A Spitzer-selected Galaxy Cluster at $\mathrm{z}=1.62$. Astrophys. J. 716, 15031513 (2010). 1002.3158 .

106. Gonzalez, A. H. et al. The Massive and Distant Clusters of WISE Survey. I. Survey Overview and a Catalog of $>2000$ Galaxy Clusters at $z \simeq 1$. Astrophys. J. Suppl. 240, 33 (2019). 1809.06820 .

107. Wilson, G. et al. Spectroscopic Confirmation of a Massive Red-Sequence-Selected Galaxy Cluster at $\mathrm{z}=1.34$ in the SpARCS-South Cluster Survey. Astrophys. J. 698, 1943-1950 (2009). 0810.0036.

108. Muzzin, A. et al. Spectroscopic Confirmation of Two Massive Red-Sequence-Selected Galaxy Clusters at $z \sim 1.2$ in the SpARCS-North Cluster Survey. Astrophys. J. 698, 19341942 (2009). 0810.0005 .

109. Sawicki, M. The 1.6 Micron Bump as a Photometric Redshift Indicator. Astron. J. 124, 3050-3060 (2002). astro-ph/0209437.

110. Stanford, S. A. et al. IDCS J1426.5+3508: Discovery of a Massive, Infrared-selected Galaxy Cluster at $\mathrm{z}=1.75$. Astrophys. J. 753, 164 (2012). 1205.3786.

111. Rettura, A. et al. Candidate Clusters of Galaxies at $z>1.3$ Identified in the Spitzer South Pole Telescope Deep Field Survey. Astrophys. J. 797, 109 (2014). 1404.0023.

112. Ashby, M. L. N. et al. SEDS: The Spitzer Extended Deep Survey. Survey Design, Photometry, and Deep IRAC Source Counts. Astrophys. J. 769, 80 (2013). 
113. Brodwin, M. et al. The Era of Star Formation in Galaxy Clusters. Astrophys. J. 779, 138 (2013). 1310.6039 .

114. Nantais, J. B. et al. Stellar mass function of cluster galaxies at $z \sim 1.5$ : evidence for reduced quenching efficiency at high redshift. Astron. Astrophys. 592, A161 (2016). 1606.07832.

115. Bruzual, G. \& Charlot, S. Stellar population synthesis at the resolution of 2003. Mon. Not. R. Astron. Soc. 344, 1000-1028 (2003). arXiv:astro-ph/0309134. 


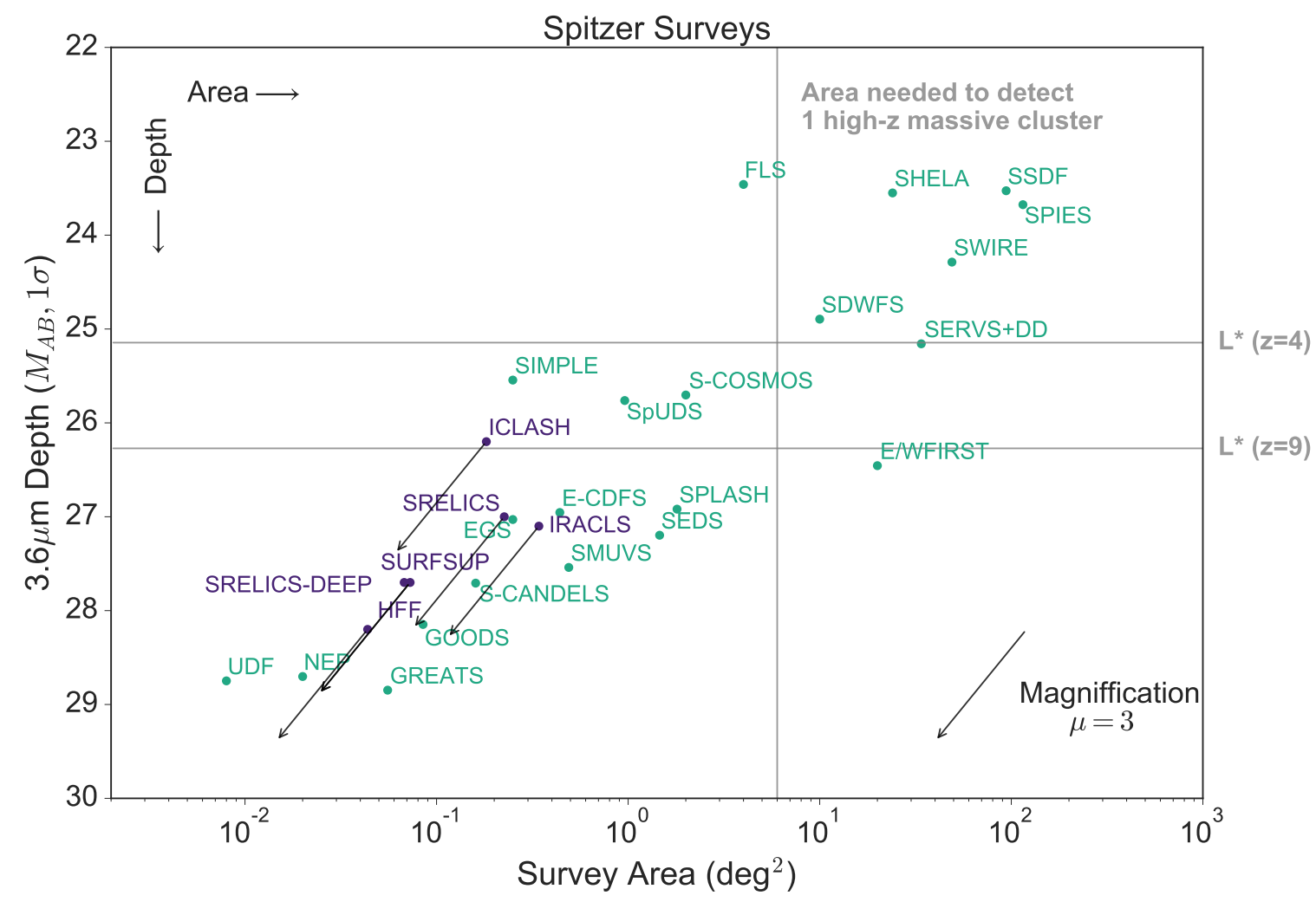

Figure 1: Sensitivities and area of Spitzer Surveys. The $3.6 \mu \mathrm{m} \mathrm{1-} \sigma$ point-source sensitivities vs area surveyed for surveys executed during the Spitzer mission. Recent Spitzer Surveys have reached the depths that allow us to push observations to observe typical $\left(L^{*}\right)$ galaxies at e.g., $z>4$ and $z>9$ (horizontal grey lines). Plotted are depths and areas for both field (green) and lensing (purple) surveys as symbols. The lensing surveys have a typical magnifications of $\mu \sim 3$, the effect of such a magnification (increased depth and reduced area) are indicated with arrows. The typical area needed to detect $\sim 1$ high-redshift $(z \gtrsim 1)$ massive cluster $\left(M>10^{14} M_{\odot}\right)$ is indicated by the vertical grey line. Adapted from [75]. 


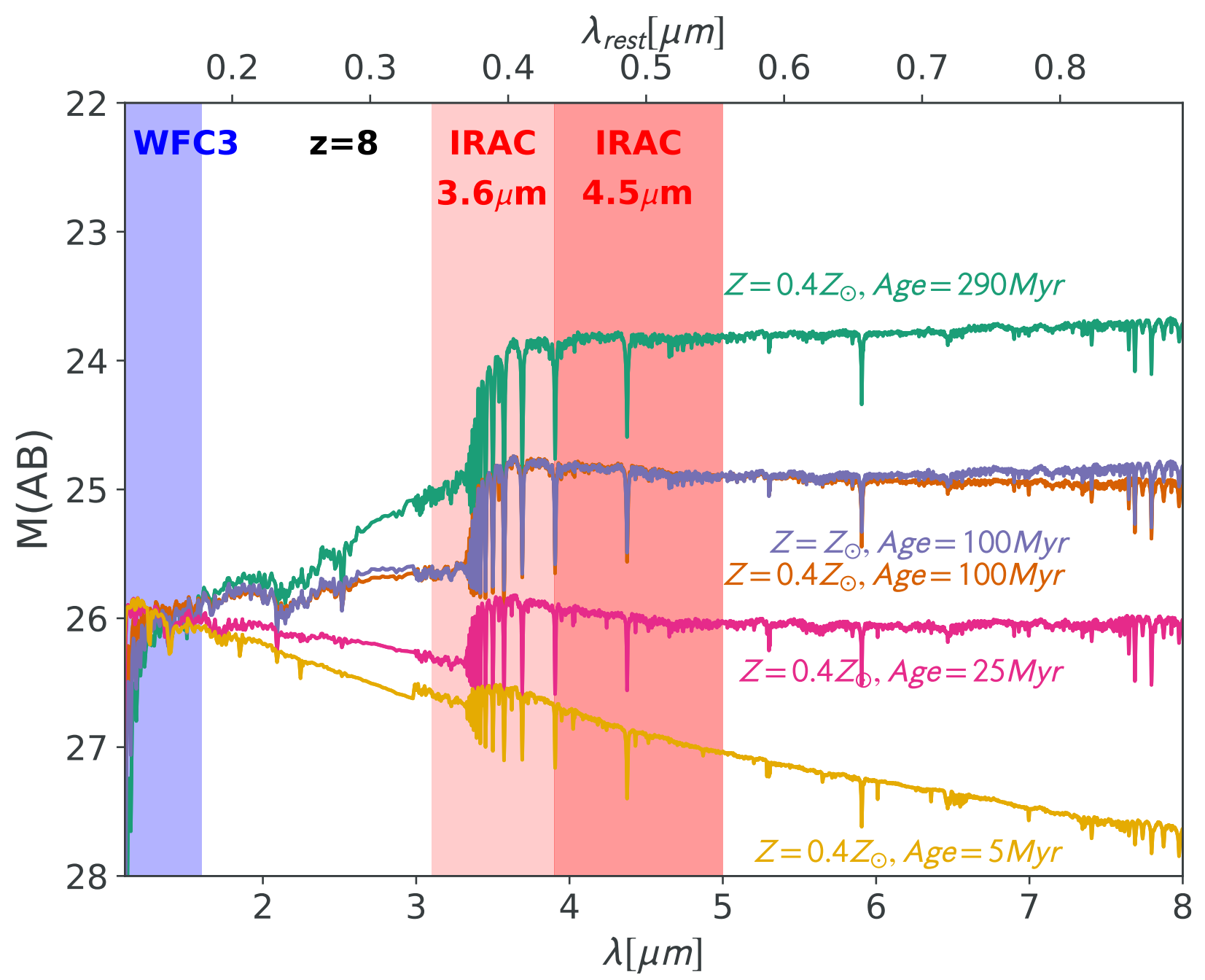

Figure 2: Spitzer data is crucial for determining stellar ages. Five different spectral energy distributions (SEDs) for starburst galaxies of different ages (from 115) redshifted to $z=8$. Whereas all these galaxies have similar colors in HST/WFC3 bands (blue shaded region) relative to typical photometric uncertainties, the different ages can be easily distinguished once $3.6 \mu \mathrm{m}$ and $4.5 \mu \mathrm{m}$ Spitzer/IRAC imaging is added (the $3.6 \mu \mathrm{m}$ and $4.5 \mu \mathrm{m}$ IRAC bands are shown with the light and dark red shaded regions), as their $m_{H_{160 \mathrm{w}}}-m_{3.6 \mu \mathrm{m}}$ and $m_{H_{160 \mathrm{w}}}-m_{4.5 \mu \mathrm{m}}$ colors are very different. We plot a $Z=0.4 Z_{\odot}$ starburst galaxy SED with stellar population at $t=290 \mathrm{Myr}$ after the burst (green), $100 \mathrm{Myr}$ (orange), $25 \mathrm{Myr}$ (pink) and $5 \mathrm{Myr}$ (yellow). Also shown is a $t=100 \mathrm{Myr}$ SED with $Z=Z_{\odot}$ (purple), to show the effect of metâlicity degeneracy with age, which is small. 


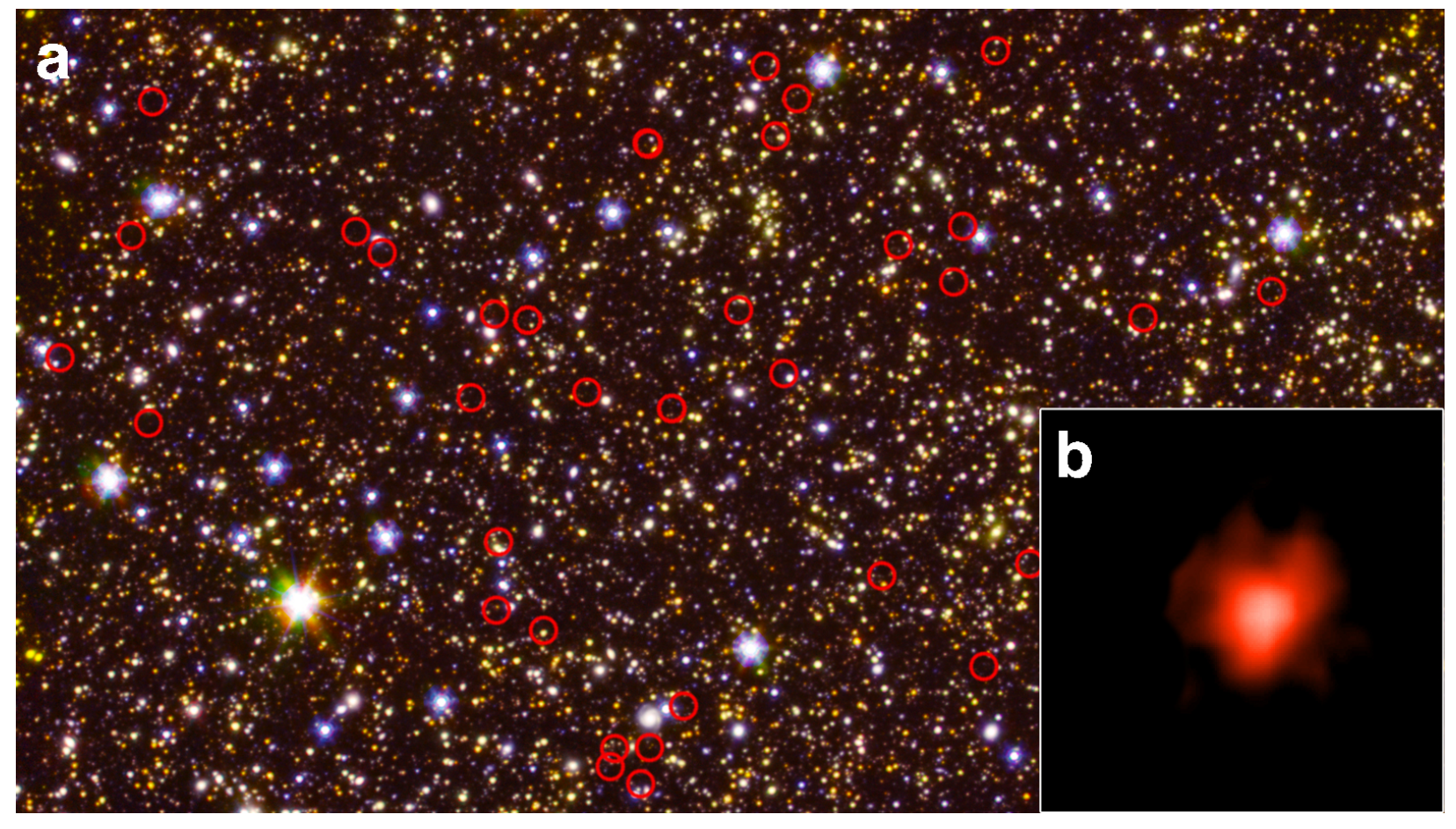

Figure 3: High-redshift galaxies detected in Spitzer images. (a) Color composite image of the central deepest region of the GREATS (GOODS Re-ionization Era wide-Area Treasury). A subset of $z \sim 8$ galaxies from [43] are circled in red. (b) An example of such a galaxy is shown in the inset. Image credit: NASA/JPL-Caltech/ESA/Spitzer/P. Oesch/S. De Barros/ I.Labbe. 


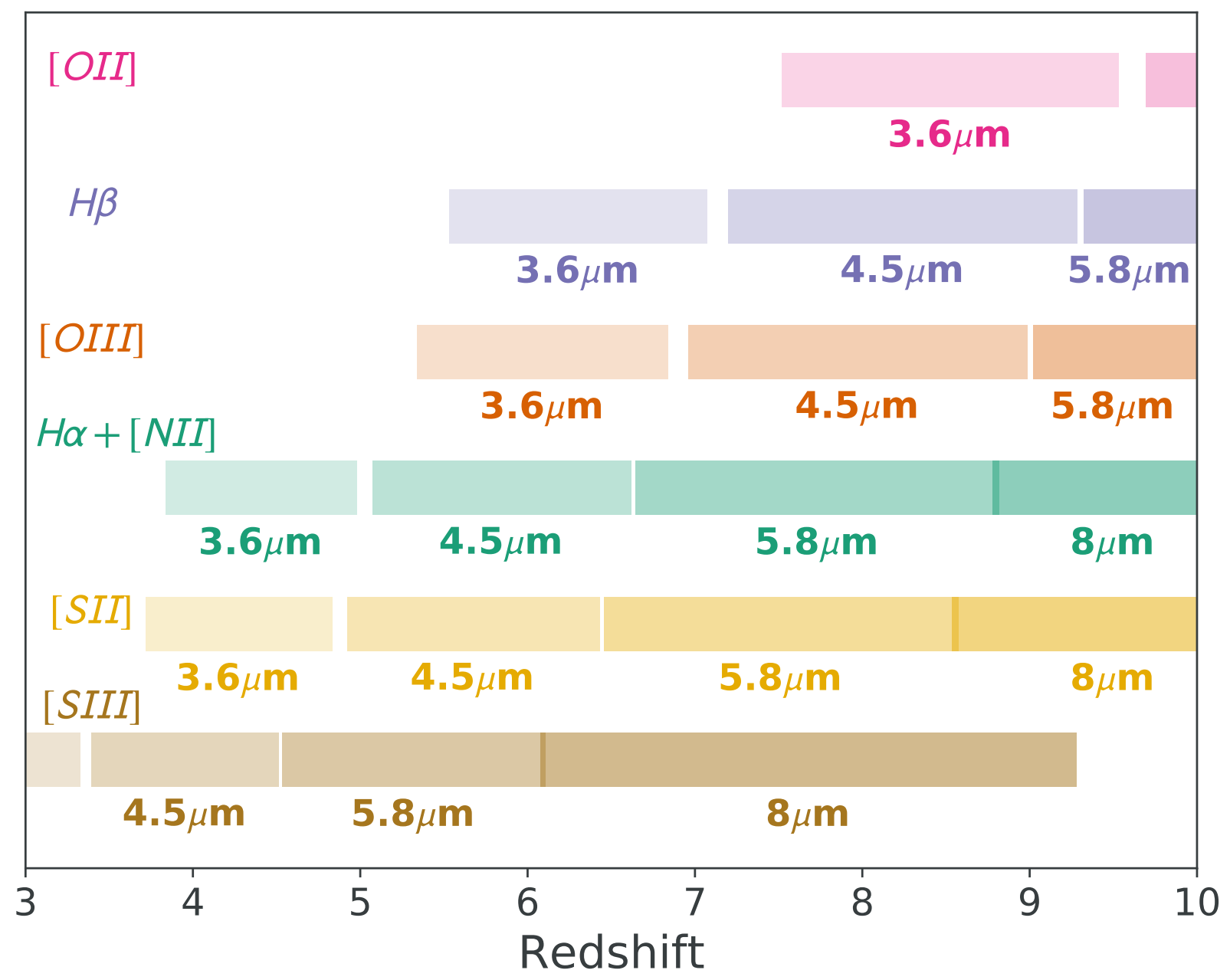

Figure 4: Spitzer data can be used to measure (combined) strengths of several prominent optical lines. Lines visible in different Spitzer channels are plotted as a function of redshift. 

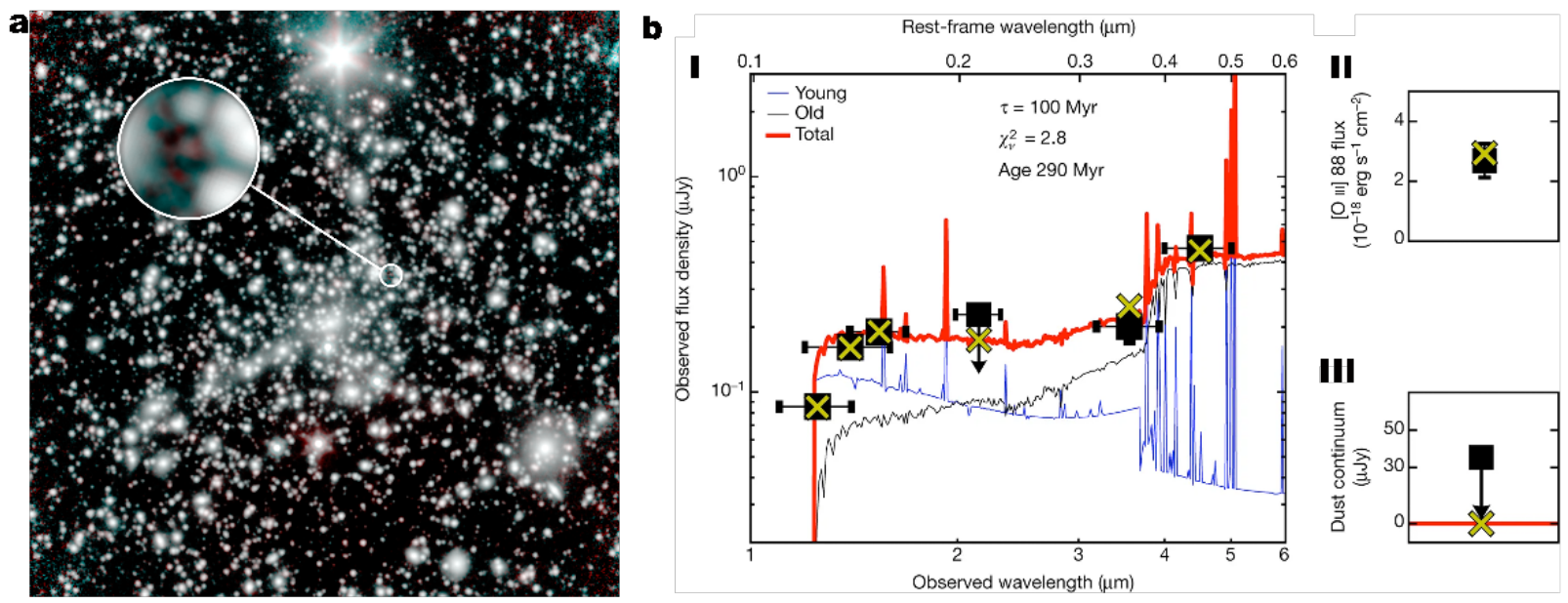

Figure 5: MACS1149-JD is the best example with an evidence of old stellar population at $z \sim 9$. a Spitzer image of MACS1149-JD from SURFS UP survey. MACS1149-JD was first discovered by [55] and first detected in both $3.6 \mu \mathrm{m}$ and $4.5 \mu \mathrm{m}$ in SURFS UP survey (26, image from Spitzer press release feature14-13). b SED modeling of the object. SED modeling provided an evidence of old stellar population once the redshift was determined to be at $z=9.1096 \pm 0.0006$. bI In particular, Spitzer fluxes can not be fit without an inclusion of old ( $290_{-120}^{+190} \mathrm{Myr}$ old) stellar population. Black squares show (from left to right) F125W, F140W and F160W data from HST, a $2 \sigma$ upper limit for the Ksband from VLT/HAWK-I, and $3.6 \mu \mathrm{m}$ and $4.5 \mu \mathrm{m}$ fluxes from Spitzer/IRAC. The red solid line indicates the SED model and the corresponding magnitudes are shown by yellow crosses. Blue and black lines represent the contributions from the young $\left(3_{-1}^{+2} \mathrm{Myr}\right)$ and old populations, respectively. bII, The black square is the observed [O Roman3] $88 \mu \mathrm{m}$ emission line flux and its $1 \sigma$ uncertainty, while the yellow cross indicates the model prediction. bIII, The black square shows the $2 \sigma$ upper limit for the dust continuum flux density, and the yellow cross indicates the model prediction. [53]. 

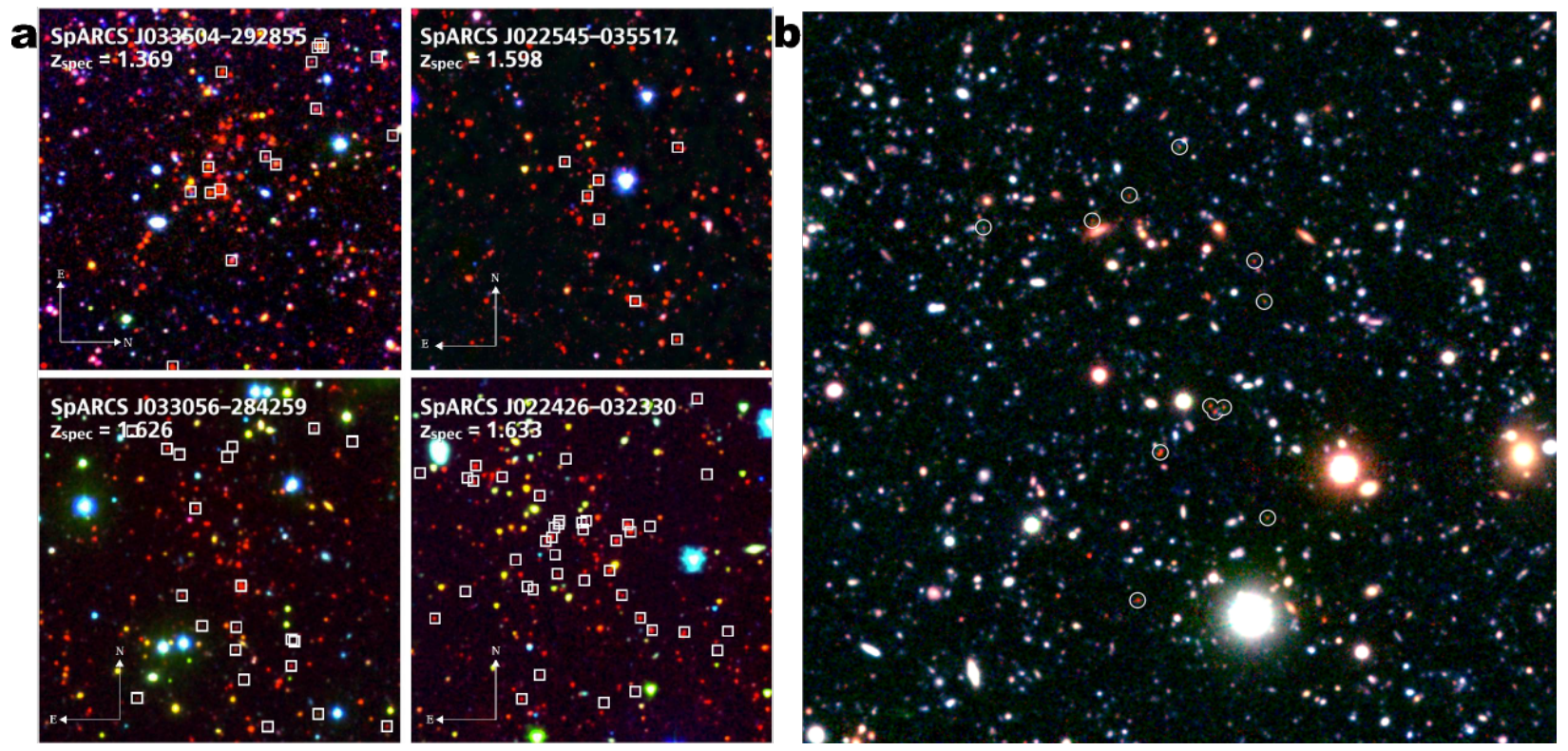

Figure 6: Spitzer data has been very effective in searching for and characterizing $z>1$ galaxy clusters and protoclusters. a Examples of high-redshift clusters from SpARCS survey.

Tri-color gz[3.6] images of the central regions of the four southern $z>1.35$ SpARCS clusters. Spectroscopic cluster members are marked with white squares [114]. b Image of a proto-cluster of galaxies at $z=5.3$. The cluster was discovered by a suite of multi-wavelength observations made with Spitzer, Chandra, HST, Subaru and Keck telescopes. Members of the developing cluster are circled in white (Spitzer press release ssc2011, 99). 